\title{
FABRY-PÉROT INTERFEROGRAMS OF THE SOLAR MgII RESONANCE LINES
}

\author{
B. BATES, D. J. BRADLEY, C. D. MCKEITH, and N. E. MCKEITH \\ Department of Pure and Applied Physics, The Queen's University of Belfast, \\ Belfast, N. Ireland \\ and \\ W. M. BURTON, H. J.B.PAXTON, D. B.SHENTON, and R. WILSON \\ Astrophysics Research Unit, Culham Laboratory, Abingdon, Berks., England
}

The resonance lines of $\mathrm{MgII}$ occur at wavelengths $(2802.7 \AA, 2795.5 \AA)$ just beyond the extinction limit of the Earth's atmosphere. At such wavelengths sophisticated optical techniques can now be employed and this fact, together with the high cosmic abundance of magnesium, makes these lines particularly important for study in UV Astronomy. In the case of the Sun, the lines consist of a broad absorption with a pronounced emission core.

The first high resolution observations $(\sim 0.03 \AA)$ were obtained by Purcell et al. (1962) using an echelle spectrometer in a Sun-pointing rocket. The resulting profiles were composite ones averaged over one-third of the solar disk, but since then spatially resolved $(\sim 10$ arc sec), high resolution $(0.045 \AA)$ spectra have been obtained with balloon borne spectrographs by Lemaire and Blamont (1967) and Lemaire (1969). These give, for the first time, information on the centre-to-limb variation of the line profiles.

The purpose of this note is to indicate some new results obtained with an optically contacted Fabry-Pérot interferometer, internally mounted in an echelle spectrograph, which was flown on a stabilised Skylark Rocket launched from the Woomera Range, South Australia at 05.35 UT on December 4, 1968. The experiment was designed to give a spectral resolution of $0.03 \AA$ with spatial resolution of 6 arc sec along a solar diameter. Forty-five interferograms were recorded producing 500 useful spectral profiles of both the MgII lines, at 15 different positions on the solar disk. The payload also included a pinhole camera assembly to record monochromatic solar images at different wavelengths, including the extreme ultraviolet.

Figure 1 shows a section of one interferogram near the MgII doublet, together with an $\mathbf{H} \alpha$ photograph taken by the Carnarvon Station, Western Australia at approximately the same time. The exact location of the slit has not yet been established from the telemetry and analysis but its approximate position is indicated. The broad absorption is particularly evident, especially towards the limb and the emission components stand out clearly. The Fraunhofer absorption lines show up as breaks in the channels.

Densitometer traces of one set of $\mathrm{Mg}_{\text {II }}$ profiles covering the emission cores and obtained by scanning along the different channels are shown in Figure 2. In general they are similar to those reported by Lemaire (1969). The central intensities of the 


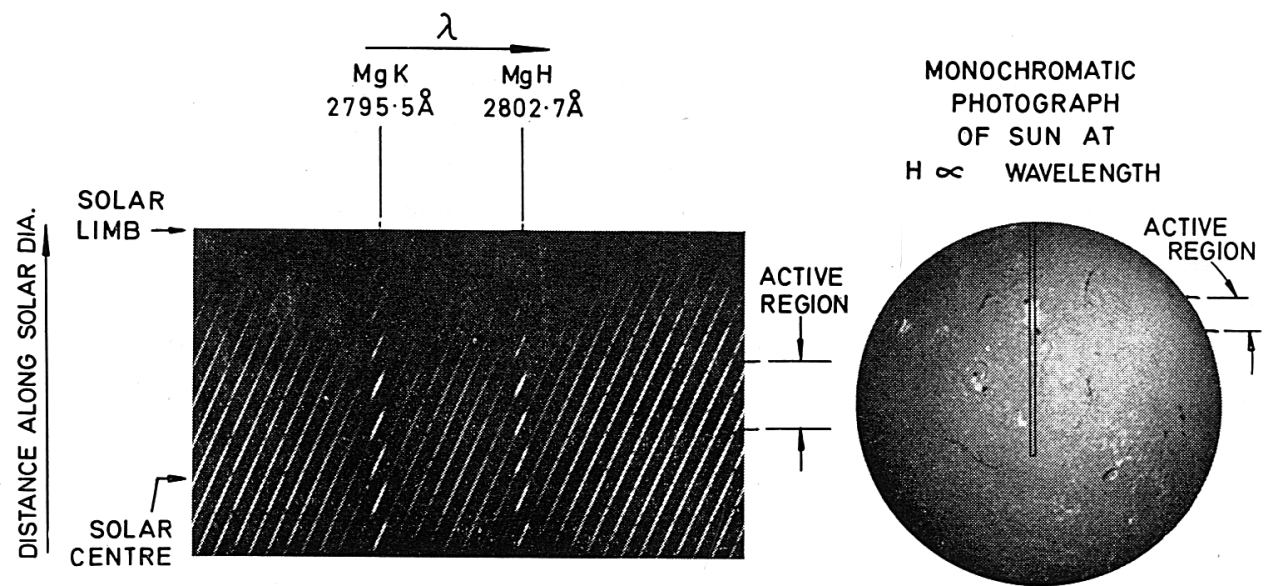

Fig. 1. Solar interferogram covering the $\mathrm{MgII} \mathrm{H}$ and $\mathrm{K}$ lines at $2802.7 \AA$ and $2795.5 \AA$. The lower limit of the fringes is set by the end of the slit which covers the solar disk and the upper limit is set by the solar limb. Also shown is a monochromatic photograph of the Sun recorded at the $\mathrm{H} \alpha$ wavelength.

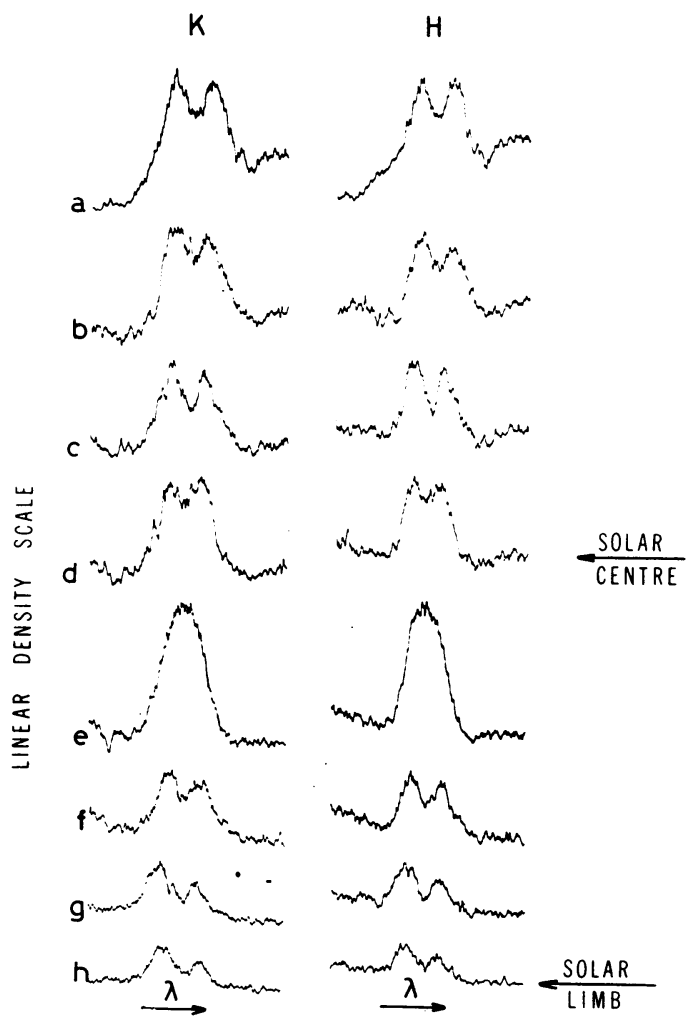

Fig. 2. A selection of microdensitometer tracings of the $\mathrm{Mg}_{\mathrm{II}} \mathrm{H}$ and $\mathrm{K}$ emission cores at various points on the solar disk. 
emission peaks decrease whereas their separation increases from centre to limb. The marked feature of the interferograms is the wide variation in profiles over the disk. In some cases, strong $\mathrm{H}_{2}$ and $\mathrm{K}_{2}$ emission lines are crossed by two absorption cores whereas in others, usually near active regions, absorption is entirely absent as in (e) of Figure 2. However, most of the observed profiles show a double emission in the core, usually unsymmetrical. The preliminary analysis of $\mathbf{H}$ line emission profiles $\left(\mathrm{H}_{2}\right)$ in all the interferograms shows that about $50 \%$ are asymmetrical with the stronger component to the violet, $10 \%$ are asymmetrical with the stronger component to the red, $30 \%$ are symmetrical and $10 \%$ show the single enhanced emission feature. The more detailed analysis including the Fraunhofer lines is now in progress.

\section{References}

Purcell, J. D., Garrett, D. L., and Tousey, R.: 1962, in Space Research, vol. III, p. 781. Lemaire, P.: 1969, Astrophys. Letters 3, 43.

Lemaire, P. and Blamont, J. E.: 1967, Astrophys. J. 150, L129. 